\title{
Maternal nutrition modifies trophoblast giant cell phenotype and fetal growth in mice
}

\author{
Adam J Watkins ${ }^{1,2}$, Emma S Lucas ${ }^{1}$, Stephanie Marfy-Smith ${ }^{1}$, Nicola Bates ${ }^{3}$, Susan J Kimber ${ }^{3}$ \\ and Tom P Fleming ${ }^{1}$ \\ ${ }^{1}$ Centre for Biological Sciences, Southampton General Hospital, University of Southampton, \\ Southampton SO16 6YD, UK, ${ }^{2}$ School of Life and Health Sciences, Aston Research Centre for Healthy Ageing, \\ Aston University, Birmingham B4 7ET, UK and ${ }^{3}$ Faculty of Life Sciences, University of Manchester, \\ Michael Smith Building, Oxford Road, Manchester M13 9PT, UK \\ Correspondence should be addressed to A J Watkins; Email: a.watkins1@aston.ac.uk
}

\begin{abstract}
Mammalian placentation is dependent upon the action of trophoblast cells at the time of implantation. Appropriate fetal growth, regulated by maternal nutrition and nutrient transport across the placenta, is a critical factor for adult offspring long-term health. We have demonstrated that a mouse maternal low-protein diet (LPD) fed exclusively during preimplantation development (Emb-LPD) increases offspring growth but programmes adult cardiovascular and metabolic disease. In this study, we investigate the impact of maternal nutrition on post-implantation trophoblast phenotype and fetal growth. Ectoplacental cone explants were isolated at day 8 of gestation from female mice fed either normal protein diet (NPD: 18\% casein), LPD (9\% casein) or Emb-LPD and cultured in vitro. We observed enhanced spreading and cell division within proliferative and secondary trophoblast giant cells (TGCs) emerging from explants isolated from LPD-fed females when compared with NPD and Emb-LPD explants after 24 and 48 h. Moreover, both LPD and Emb-LPD explants showed substantial expansion of TGC area during 24-48 h, not observed in NPD. No difference in invasive capacity was observed between treatments using Matrigel transwell migration assays. At day 17 of gestation, LPD- and Emb-LPD-fed conceptuses displayed smaller placentas and larger fetuses respectively, resulting in increased fetal:placental ratios in both groups compared with NPD conceptuses. Analysis of placental and yolk sac nutrient signalling within the mammalian target of rapamycin complex 1 pathway revealed similar levels of total and phosphorylated downstream targets across groups. These data demonstrate that early post-implantation embryos modify trophoblast phenotype to regulate fetal growth under conditions of poor maternal nutrition. Reproduction (2015) 149 563-575
\end{abstract}

\section{Introduction}

The development of the mammalian fetus is fundamentally dependent upon successful implantation of the embryo into the uterine tissue. This essential step is regulated by the action of the trophoblast cells that coordinate the complex processes of embryo attachment and implantation, and uterine spiral artery remodelling, and initiate the development of the placenta. At the time of implantation, the embryo comprises two distinct cell lineages, the inner cell mass (ICM) containing the progenitor cells of the fetal and primitive endoderm lineages, and the trophectoderm (TE) comprising the progenitors of the chorioallantoic placenta. Following implantation, TE cells not in direct contact with the ICM (mural TE) exit mitosis, endo-reduplicate their DNA and give rise to the primary trophoblast giant cells (TGCs). These primary TGCs form blood sinuses at the periphery of the embryo, contributing to the provision of nutrients and oxygen to the developing embryo by the yolk sac
(Bevilacqua \& Abrahamsohn 1988). In contrast, the TE cells directly overlying the ICM (polar TE) differentiate into the chorion and ectoplacental cone (EPC), from which secondary TGCs are derived (Simmons \& Cross 2005).

The large polyploid secondary TGCs invade into the maternal decidual tissue and spiral arteries to establish maternal-fetal nutrient transfer (Adamson et al. 2002) as well as secreting a range of hormones and cytokines that modulate the maternal uterine environment, both essential for fetal growth (Simmons et al. 2007, Soares et al. 2007). At around day 8.5 of gestation in the mouse (E8.5), the allantois begins to attach to the chorion initiating the formation of both the junctional and labyrinth zones of the developing placenta. Ultimately, the labyrinth zone will mediate nutrient exchange between the fetus and mother through a highly developed vascular network, while the junctional zone becomes the site of growth factor and pregnancy-related hormone production (Georgiades et al. 2002). Therefore, 
disruption in the normal process of trophoblast differentiation or phenotype specification during early gestation (E8.5) is likely to affect detrimentally embryo viability, placental morphology and function and ultimately fetal growth and development.

Over recent decades, the regulation of fetal growth and development has gained significant interest in light of human epidemiological and animal model observations, demonstrating links between altered fetal growth and predisposition to adult-onset cardiovascular and metabolic diseases (Hanson \& Gluckman 2014). The significant similarities between human and rodent preimplantation embryo development, implantation and placental morphology mean that mice and rats provide biologically applicable models with which to investigate the sensitivity of the developing placenta and its impact on offspring health. In mice, maternal caloric restriction results in differential placental expression of genes associated with intra-uterine growth restriction and genome-wide hypomethylation (Chen et al. 2013), while a high-fat diet induces sex-specific changes in placental gene expression for immune and inflammatory responses and regulators of epigenetic modifications (Gabory et al. 2012). Changes in placental gene expression within apoptosis, growth inhibition and epigenetic modification pathways have also been observed in response to a maternal low-protein diet (LPD) in mice (Gheorghe et al. 2009), while, in the rat, changes in placental mammalian target of rapamycin complex 1 (mTORC1) and amino acid transporter protein levels have been demonstrated (Rosario et al. 2011). Additional changes in placental structure, nutrient transport and fetal cytokine levels have also been reported in response to maternal dietary manipulation (Armitage et al. 2004, Coan et al. 2011, Chen et al. 2013, Sferruzzi-Perri et al. 2013, Kim et al. 2014).

We have demonstrated that a maternal LPD ( $9 \%$ casein) fed to mouse dams exclusively during preimplantation development (days 0-3.5 of gestation; Emb-LPD) enhances postnatal growth and adiposity and induces hypertension, vascular dysfunction and altered behavioural characteristics in adult offspring (Watkins et al. 2008, 2010, 2011). We observed additionally that enhanced early postnatal growth was a significant predictor of adult adiposity, cardiovascular health and behaviour (Watkins et al. 2008). Blastocysts transferred from LPD-fed dams into recipient NPD-fed dams displayed elevated fetal growth, when assessed at E17, demonstrating that offspring programming is initiated during preimplantation development (Watkins et al. 2008). Analysis of embryonic and extraembryonic lineage tissues from Emb-LPD-fed dams has revealed elevated blastocyst TE:ICM ratio and differential signalling through the mTORC1 nutrient sensing pathway (Eckert et al. 2012), enhanced endocytic and lysosomal activity within blastocyst TE and embryoid body primitive endoderm (Sun etal. 2014), and enhanced uptake and transport capacity within the late gestation visceral yolk sac (Watkins et al. 2008). From these data, we propose that the preimplantation embryo senses the maternal uterine nutritional status directly and initiates a series of adaptive mechanisms within the extraembryonic lineages to enhance nutrient uptake and maintain growth. However, upon restoration of optimal maternal nutrition postimplantation, these enhanced nutrient uptake adaptations become maladaptive, promoting increased fetal growth and adult offspring ill-health.

While the impact of maternal diet on embryonic and late gestation placental nutrition-uptake mechanisms has been investigated in detail, the nutritional impacts on early post-implantation dynamics, and how these processes influence the development of the mature placenta, remain unknown. Therefore, in the current study, we have extended our mouse gestational dietary model to investigate for the first time the impact of EmbLPD on early post-implantation TGC phenotype in vitro, late gestation fetal growth and placental development.

\section{Materials and methods}

\section{Animal treatments}

All mice and experimental procedures were conducted using protocols approved by, and in accordance with, the UK Home Office Animal (Scientific Procedures) Act 1986 and local ethics committee at the University of Southampton. Virgin female MF-1 mice (aged 7-8.5 weeks), derived within the University's Biomedical Research Facility, were maintained on a $0700 \mathrm{~h}$ light:1900 h darkness cycle at a temperature of $20-22{ }^{\circ} \mathrm{C}$ and fed on standard laboratory chow ad libitum (Special Diet Services Ltd, Witham, Essex, UK), and were housed singly overnight with MF-1 studs. The presence of a vaginal plug the following morning was taken as a sign of mating. Plug-positive females were housed singly and allocated randomly to one of the three isocaloric (calories/g; Special Dietary Services Ltd; composition published previously (Watkins et al. 2011)) dietary regimens; i) normal protein diet (NPD; 18\% casein, $42.5 \%$ maize starch, $21.3 \%$ sucrose, $10 \%$ corn oil, $5 \%$ cellulose), ii) LPD (9\% casein, $48.5 \%$ maize starch, $24.3 \%$ sucrose, $10 \%$ corn oil, $5 \%$ cellulose) or iii) LPD from detection of a vaginal plug until day 3.5 of gestation, then switched to NPD for the remaining days of gestation (Emb-LPD). Females were culled via cervical dislocation at either E8.5, for the isolation and culture of post-implantation EPCs, or at day 17 (E17) for analysis of fetal growth and placental and yolk sac mTORC1 signalling.

\section{Isolation of EPC explants}

In the mouse, E8.5 represents a developmental stage during which trophoblast differentiation and early placental lineage induction are being established. Therefore, analysis of EPC outgrowth phenotype at this stage could provide significant insights into the establishment of the mature placenta and the regulation of fetal growth in response to maternal diet (El-Hashash \& Kimber 2004, El-Hashash et al. 2005). At E8.5, NPD-, LPD- and Emb-LPD-fed dams were culled by cervical dislocation. Whole uteri were excised and placed within 
pre-warmed $\left(37^{\circ} \mathrm{C}\right)$ DMEM (Life Technologies) with $10 \%(\mathrm{v} / \mathrm{v})$ heat-inactivated FCS (Sigma-Aldrich). Individual decidual capsules were dissected from both uterine horns before isolation of whole embryos from within the surrounding decidual tissue. EPCs were separated from the embryo at the junction with the extraembryonic ectoderm using sterile fine forceps.

\section{Culture and outgrowth of secondary TGCs}

Previously, we have demonstrated increased TE cell number and in vitro outgrowth expansion in blastocysts collected from LPD-fed dams (Eckert et al. 2012). To determine whether similar increases in expansive phenotype were still present within EPC outgrowths at E8.5, we cultured explants on coverslips to assess their growth within a two-dimensional plane. This approach enabled us to determine migratory phenotype while maintaining the close architectural structure of the outgrowth and preserving cell-cell contacts. Sterile coverslips were coated with BD Matrigel basement membrane matrix (BD Biosciences, Oxford, UK) diluted to $6 \mathrm{mg} / \mathrm{ml}$ in RPMI 1640 medium (Life Technologies) and left at $4{ }^{\circ} \mathrm{C}$ overnight in four-well plates (Sigma-Aldrich). The following morning, the Matrigel-RPMI medium was replaced with $500 \mu$ l sterile filtered RPMI 1640 medium (Life Technologies) containing 2\% KnockOut Serum Replacement (Life Technologies), penicillin-streptomycinglutamine mix (Life Technologies) and $26.2 \mu \mathrm{mol}$ twomercaptoethanol (Sigma-Aldrich) and allowed to equilibrate at $37^{\circ} \mathrm{C}$ in an atmosphere of $5 \% \mathrm{CO}_{2}$ for at least $1 \mathrm{~h}$. Dissected EPC explants were cultured individually at $37{ }^{\circ} \mathrm{C}$ in an atmosphere of $5 \% \mathrm{CO}_{2}$ for up to $48 \mathrm{~h}$ with a $50 \%$ medium change after $24 \mathrm{~h}$.

\section{Assessment of EPC explant invasive capacity}

In addition to cell migration, cell invasion is an important event in the development of the placenta. To obtain a greater understanding of the invasive capacity of outgrowths, we cultured EPC explants on Matrigel transwell inserts. Using this approach, we could quantitate invasive and migratory capacity through a three-dimensional membrane, replicating more closely an in vivo environment when compared with coverslips. Matrigel invasion assays of EPC explants were performed using BD BioCoat Matrigel Invasion Chambers (VWR, Lutterworth, UK). Transwell inserts ( $8 \mu \mathrm{m}$ pore size) were re-hydrated within a 24-well plate with RPMI 1640 medium (Life Technologies) (500 $\mu \mathrm{l} /$ well and per insert) at $37{ }^{\circ} \mathrm{C}$ in an atmosphere of $5 \% \mathrm{CO}_{2}$ for $2 \mathrm{~h}$ after which the medium was replaced with RPMI medium containing serum replacement, penicillinstreptomycin-glutamine and two-mercaptoethanol (as mentioned earlier in this study; $750 \mu \mathrm{l}$ within the well and $500 \mu \mathrm{l}$ within the invasion chamber). Invasion chambers were allowed to equilibrate at $37^{\circ} \mathrm{C}$ in an atmosphere of $5 \% \mathrm{CO}_{2}$ for at least $1 \mathrm{~h}$. EPC explants, isolated as described earlier in this study, were cultured singly per well for up to $48 \mathrm{~h}$ with a $50 \%$ medium change after $24 \mathrm{~h}$.

\section{Morphological and invasive analysis of outgrowths}

EPC outgrowths, both on coverslips and invasion chambers, were fixed after 24 or $48 \mathrm{~h}$ of culture in $4 \%$ neutral buffered formalin (Sigma-Aldrich) for 15 min before permeabilisation with $0.1 \%$ Triton-X100 (Sigma-Aldrich) for $5 \mathrm{~min}$, and washed three times with PBS at room temperature. Background staining was minimised with ammonium chloride $(2.6 \mathrm{mg} / \mathrm{ml}$ in PBS) for 10 min before an additional 5 min permeabilisation step $(0.1 \%$ Triton-X100) and PBS washing, all conducted at room temperature. Non-specific binding was blocked with $2 \%$ BSA in PBS containing $0.1 \%$ Triton-X100 for $30 \mathrm{~min}$ at room temperature before overnight incubation at $4{ }^{\circ} \mathrm{C}$ with a primary antibody for $\alpha$-tubulin (1:2000; Cell Signaling, Danvers, MA, USA) in PBS with $1 \%$ BSA and $0.1 \%$ Triton-X100. Following washing in PBS with $0.1 \%$ Triton-X100, outgrowths were incubated with the appropriate Alexa Fluor conjugated secondary antibody (1:10 000; Molecular Probes, Life Technologies, Paisley, UK) for $1 \mathrm{~h}$ at room temperature and counterstained for nuclei using DAPI (Sigma-Aldrich) for 10 min before mounting on slides in DPX (Fisher, Loughborough, UK). All outgrowths were imaged using a Leica DSM 5000 microscope with epifluorescence capacity and analysed using the Volocity Software. The central EPC was defined as the central, single mass within which individual cell nuclei could not be determined. Proliferative trophoblast nuclei were defined as those located within the proximity of the central EPC and having a nuclear area $<300 \mu^{2}$ (Scott et al. 2000, El-Hashash \& Kimber 2004). Secondary trophoblasts were defined as those located at the periphery of the outgrowth and possessing a nuclear area $>300 \mu \mathrm{m}^{2}$ (El-Hashash \& Kimber 2004). Each outgrowth was assessed for the area of the EPC, proliferative and secondary trophoblast cells and of the whole outgrowth; distance of the furthest nucleus from the centre of the EPC; and perimeter of the outgrowth and number of secondary trophoblast cells.

\section{Analysis of fetal growth}

At E17, NPD-, LPD- and Emb-LPD-fed dams were culled for the analysis of fetal growth. Whole uteri were removed, placed in ice-cold PBS and the number of conceptuses per horn recorded. Individual conseptuses were dissected carefully from the uterine tissue and weighed before separation of the fetus from the placenta and yolk sac. Each fetus, placenta and yolk sac were weighed individually before decapitation of the fetus for the collection of serum. Isolated placentae and yolk sacs were snap frozen in liquid nitrogen ahead of protein level analysis.

\section{Placental and yolk sac mTOR analysis}

Individual placentae and yolk sacs were homogenised in lysis buffer (50 mM HEPES pH 7.4, $150 \mathrm{mM} \mathrm{NaCl,} 1 \mathrm{mM}$ EDTA, $1 \mathrm{mM}$ EGTA, 1\% NP40; Sigma-Aldrich) containing 2\% v/v protease inhibitor (complete mini protease inhibitor cocktail, Roche) and $1 \% \mathrm{v} / \mathrm{v}$ phosphatase inhibitor cocktails 1 and 2 (Sigma-Aldrich). Protein levels were determined using the DC protein assay kit (Bio-Rad) before boiling in LDS sample buffer (Life Technologies) and running on $4-12 \%$ bis-tris gels (Life Technologies). Proteins were transferred onto nitrocellulose membranes overnight, blocked in 5\% milk in TBS-Tween (0.1\% Tween 20; Sigma-Aldrich) before incubation overnight at $4{ }^{\circ} \mathrm{C}$ with primary antibodies (Cell Signaling) for total 4E-BP1 (1:1000), phospho- (Thr37/46) 4E-BP1 (1:1000), total S6 
ribosomal protein (1:2000), phospho- (Ser235/236) S6 ribosomal protein $(1: 2000)$ and $\alpha$-tubulin $(1: 10000)$, all diluted in $5 \%$ BSA in TBS-Tween. After overnight incubation, membranes were washed in TBS-Tween before $1 \mathrm{~h}$ of incubation with appropriate species-specific, IRDye-conjugated secondary antibodies (Rockland, Limerick, PA, USA) in 5\% milk in TBSTween 20 at room temperature. Following additional washes with TBS-Tween20, membranes were analysed by densitometry using an Odyssey Infrared Imaging System (Licor, Lincoln, NE, USA). Proteins of interest were normalised to $\alpha$-tubulin to control for differences in total protein loaded.

\section{Statistical analysis}

All data were assessed for normality using the Shapiro-Wilk normality test (SPSS version 21). All fetal offspring organ weight data were analysed using a multilevel random effects regression model (SPSS version 21) to account for maternal origin of litter, gestational litter size, offspring sex and body weight where appropriate (Watkins et al. 2008). Analysis of placental and yolk sac protein levels and all EPC outgrowth measurements was performed by univariate ANOVA with Tukey's multiple comparison post hoc test for normally distributed data, or a Kruskal-Wallis test with Dunn's multiple comparisons post hoc test for non-normally distributed data (SPSS version 21). All data were assessed for interactions among maternal diet, offspring sex and litter size, where possible. Significance was considered at $P<0.05$.

\section{Results}

\section{Characterisation of EPC explant outgrowth on Matrigel-coated coverslips}

At the time of cull (E8.5), the mean number of implantation sites per female $(10.53 \pm 0.39)$ and the mean cross-sectional area of EPCs isolated for culture either on coverslips or in transwell inserts $(0.83 \pm$ $0.04 \mathrm{~mm}^{2}$ ) did not differ between treatment groups $(P>0.2)$. We first assessed the outgrowth characteristics of isolated EPC explants on coverslips coated with the basement membrane matrix Matrigel. After $24 \mathrm{~h}$ in culture, explants were observed to form a diffuse outgrowth, radiating in all directions from the central EPC. Visual inspection of outgrowths revealed them to be comprising two populations of cells; those immediately surrounding the central EPC, which appeared small, densely packed and with elongated nuclei, and those at the periphery, which were larger and possessed more rounded nuclei (Fig. 1a). After 48 h, outgrowths had increased in size but still comprised a central EPC surrounded immediately by small densely packed cells with larger cells populating the periphery (Fig. 1, b, c and d). Closer examination of these cell populations revealed the outer cells to be large $\left(>1000 \mu \mathrm{m}^{2}\right)$, flat and multinucleated, while those cells surrounding the central EPC appeared smaller, multi-layered and possessed a single nucleus (Fig. 1e and f). Previous studies have identified the cells closest to the central EPC explant as being proliferative stem cells (Scott et al. 2000, El-Hashash \& Kimber 2004), while those cells located at the outgrowth periphery display morphological characteristics of TGCs (large, multinucleated and single layered) and express specific markers such as PL-II, not expressed within proliferating cells (El-Hashash \& Kimber 2004).

Using these morphological characteristics, we defined mean fluorescence intensity ( $\boldsymbol{\alpha}$-tubulin) and nucleus area (DAPI) threshold values to define the area of the central EPC (Fig. 2a). To determine the distance of the furthest nucleus from the centre of the EPC, a square boundary encompassing the entire highlighted EPC was superimposed and the centre of this square determined by two diagonal lines connecting each corner. From the crossing point of these two lines, the distance of the furthest nuclei could be determined (Fig. 2b). In addition, we determined the area of proliferative (Fig. 2c) and secondary TGCs as well as the total area and perimeter of each outgrowth. These standardised threshold values were then applied to each outgrowth image to provide an objective set of measurement criteria. Analysis of EPC explants after $24 \mathrm{~h}$ revealed that LPD outgrowths had a significantly larger mean total area (i.e. comprising central EPC and surrounding proliferative and secondary TGCs) than NPD outgrowths $(P=0.05)$ and a trend to exceed that of Emb-LPD outgrowths ( $P=0.092)$ (Fig. $2 \mathrm{~d}$ ). Both LPD and Emb-LPD outgrowths had significantly increased mean EPC area after $24 \mathrm{~h}$ when compared with NPD outgrowths (Fig. 2e) $(P<0.01)$. No differences in mean area of proliferative or secondary TGCs, or in their combined area, were observed between groups after $24 \mathrm{~h}$ (Fig. 2f, g and h). However, Emb-LPD outgrowths had significantly reduced mean number of secondary TGC nuclei when compared with NPD and LPD outgrowths (Fig. 2i) $(P<0.01)$. Despite no significant difference in the mean distance of the furthest cell from the centre of the EPC being observed between groups (Fig. 2j), LPD outgrowths displayed an increased outgrowth perimeter when compared with NPD and Emb-LPD outgrowths (Fig. $2 \mathrm{k})(P<0.05)$.

After $48 \mathrm{~h}$ of culture on Matrigel-coated coverslips, LPD outgrowths displayed significantly elevated mean total outgrowth area (Fig. 2d), EPC area (Fig. 2e), area of secondary TGCs (Fig. 2g), combined proliferative and secondary TGC area (Fig. $2 \mathrm{~h}$ ), number of secondary TGC nuclei (Fig. 2i), distance of the furthest nucleus from the EPC centre (Fig. 2j) and outgrowth perimeter (Fig. 2k) when compared with outgrowths from both NPD- and Emb-LPD-fed females $(P<0.05)$. Outgrowths from Emb-LPD-fed females displayed a significantly reduced mean area of proliferative cells when compared with outgrowths from NPD-fed females after $48 \mathrm{~h}$ (Fig. 2f) $(P=0.006)$ and found to be at a trend level $(P=0.074)$ when compared with LPD outgrowths.

Using data collected on outgrowth proportions for each dietary group between 24 and $48 \mathrm{~h}$ in culture, we 

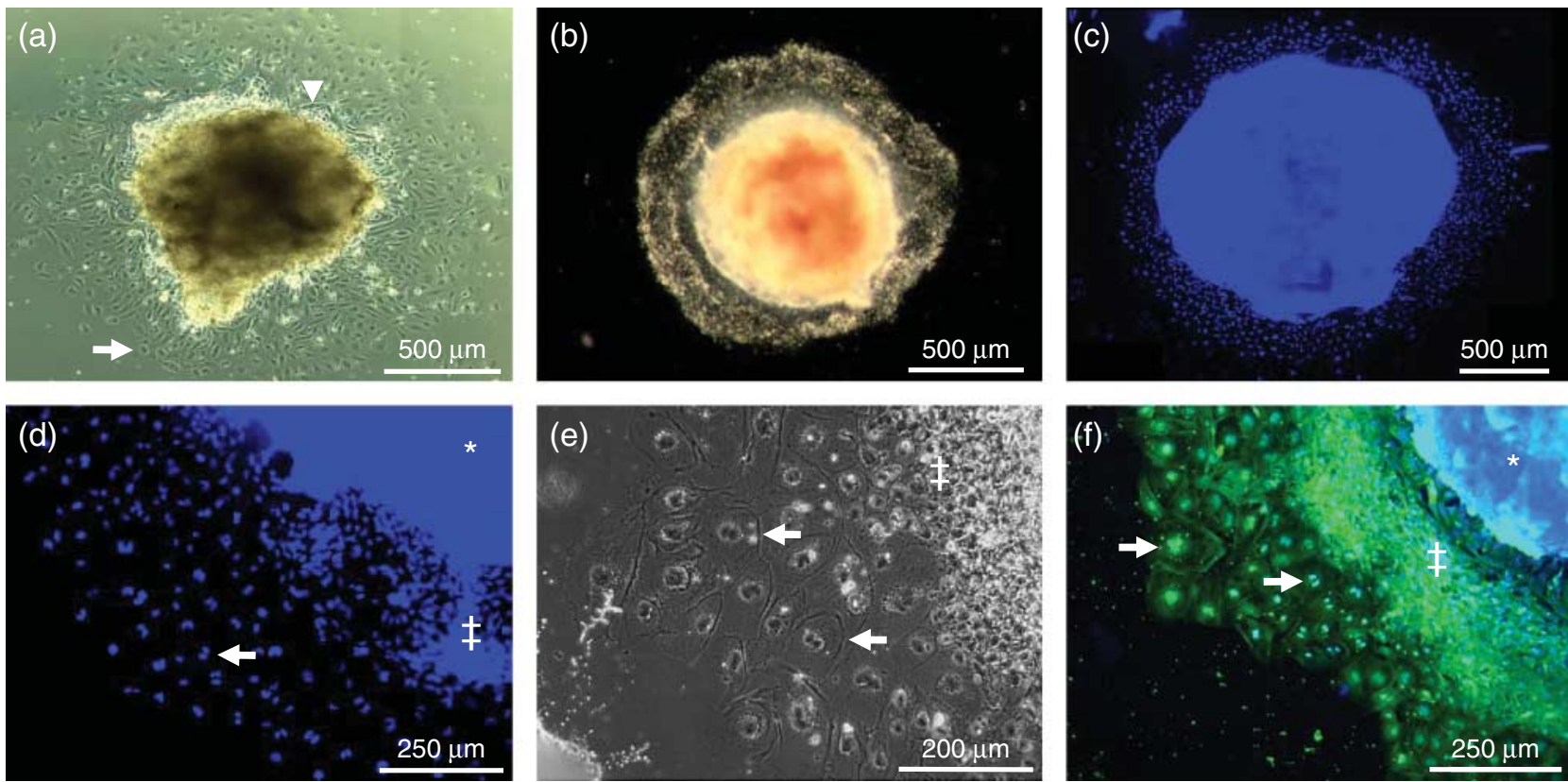

Figure 1 Representative images of E8.5 mouse EPC explant outgrowths grown on Matrigel-coated coverslips. (a) Inverted bright-field image taken under a Leica DSM 5000 microscope after $24 \mathrm{~h}$ showing small, densely packed cells and with elongated nuclei surrounding the central EPC (arrow head) and larger more widely spaced cells with rounded nuclei (arrow) at the periphery (shown at a higher magnification in $d$, e and f), or (b) topilluminated bright-field image taken under a Zeiss Stemi SV11 stereomicroscope after $48 \mathrm{~h}$ in culture. (c) Whole image of DAPI-stained outgrowth after $48 \mathrm{~h}$ in culture. (d) Magnified section of an outgrowth showing the central EPC $(*)$, small, closely associated 'proliferative' ( $\neq)$ and peripheral 'secondary' (arrow) trophoblast giant cells in outgrowths after $48 \mathrm{~h}$ in culture. (e) Phase-contrast image of peripheral trophoblast giant cells (arrow) identifying a monolayer of large, multinucleated cells after $48 \mathrm{~h}$ of culture at the periphery of the 'proliferative' ( $\ddagger$ ) cells. (f) Immunofluorescence image stained for $\alpha$-tubulin, showing multinucleated peripheral trophoblast giant cells (arrow) after $48 \mathrm{~h}$ of culture with proliferative layer ( $\neq$ ) and central EPC $\left(^{*}\right)$ shown on right.

assessed the relative development and changes in EPC, and proliferative and secondary TGC composition. After $24 \mathrm{~h}$ of culture on Matrigel-coated coverslips, outgrowths from Emb-LPD-fed females were composed of a significantly higher EPC and reduced secondary TGC proportion when compared with outgrowths from NPD-fed females (Fig. 3a) $(P<0.05)$. After 48 h of culture, no significant differences in mean EPC or secondary TGC proportions were observed between groups (Fig. 3b). However, significantly reduced proliferative TGC proportions were observed for outgrowths from females fed LPD and Emb-LPD when compared with NPD-fed females (Fig. 3b) $(P<0.05)$. This alteration in outgrowth composition between 24 and $48 \mathrm{~h}$ of culture resulted from significant reductions in mean EPC proportions (LPD $11.99 \%$, Emb-LPD $12.20 \%$ ) and increased secondary TGC proportions (LPD 14.50\%, Emb-LPD 15.49\%), while the NPD outgrowths altered their EPC and secondary TGC proportions by $1 \%$ or less (Fig. 3C).

\section{Characterisation of EPC explant invasive phenotype}

In addition to our analysis of EPC explant outgrowth on coverslips, we also assessed explant invasive capacity in vitro using Matrigel-coated transwell inserts. After $6 \mathrm{~h}$, we observed that relatively few cells had penetrated the
Matrigel coating, migrating through the $8 \mu \mathrm{m}$ pores (Fig. 4a) and spreading over the under-surface of the insert (Fig. 4b). The number of cells observed to have migrated to the under-surface of the insert increased between $12 \mathrm{~h}$ (Fig. 4c) and $24 \mathrm{~h}$ (Fig. 4d) such that, following $24 \mathrm{~h}$ in culture, a large population of cells were present on the under-surface of the insert. Closer examination of these invasive and migratory cells after $24 \mathrm{~h}$ of culture revealed a central mass of densely packed, predominately mono-nucleated cells, with a single layer of larger, multinucleated cells at the periphery (Fig. 4e and f), reflective of outgrowths cultured on coverslips. After $36 \mathrm{~h}$ of culture, the large number of cells present on the under-surface of the insert prevented identification of individual nuclei and, therefore, quantitative determination of cell migration and outgrowth invasive characteristics (data not shown). Therefore, we analysed outgrowth invasive phenotype after 18 and $24 \mathrm{~h}$ of culture, measuring the total outgrowth area and perimeter, the number of nuclei and the distance of the furthest nuclei from the centre of the outgrowth.

After $18 \mathrm{~h}$ in culture, no significant difference in the mean outgrowth area, number of nuclei, distance of the furthest nucleus, outgrowth area per nucleus or perimeter was observed between treatment groups 

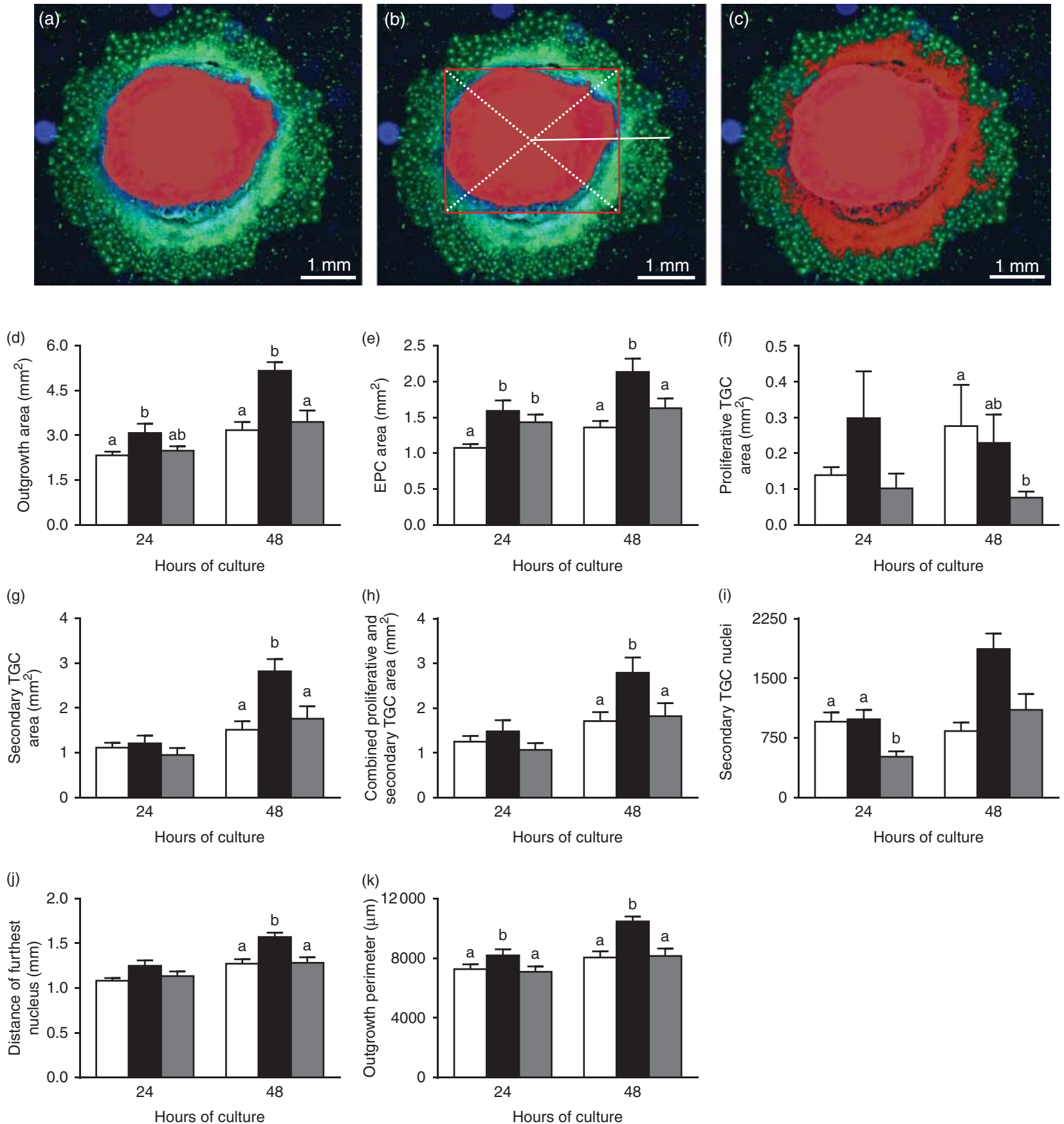

Figure 2 ( $a$, b and c) Example of E8.5 mouse EPC explant outgrowth analysis using the Volocity Software to determine EPC area (highlighted in red in a) and distance of the furthest nucleus from the EPC centre (solid white line from the centre of the superimposed square in b) and the combined area of the EPC and proliferative cells (highlighted in red) in (c). The area of the secondary TGCs was determined as the areas of the entire outgrowth minus the combined area of the EPC and proliferative TGCs. (d, e, f, g, h, i, j and k) Mean EPC outgrowth measurements from NPD (white bars), LPD (black bars) and Emb-LPD (grey bars) explants grown on Matrigel-coated coverslips after 24 and $48 \mathrm{~h} . n=10-14$ outgrowths taken from 6-8 separate females per diet per culture time and treatment group. Error bars are s.E.M. Different letters denote statistical significance between groups at $P<0.05$.

(Fig. 4g, h, i, j and k). After $24 \mathrm{~h}$ in culture, Emb-LPD outgrowths displayed a significantly reduced mean distance of the furthest nucleus from the outgrowth centre (Fig. $4 \mathrm{i} ; P=0.017$ ) and reduced mean outgrowth area and perimeter at trend levels (Fig. $4 \mathrm{~g}$ and e; $P<0.01$ ).

\section{Fetal development at E17}

Previously, we have demonstrated elevated fetal growth at E17 in offspring derived from blastocysts transferred from LPD-fed dams into NPD-fed dams (Watkins et al. 2008). This significantly increased growth reflected the 
(a)

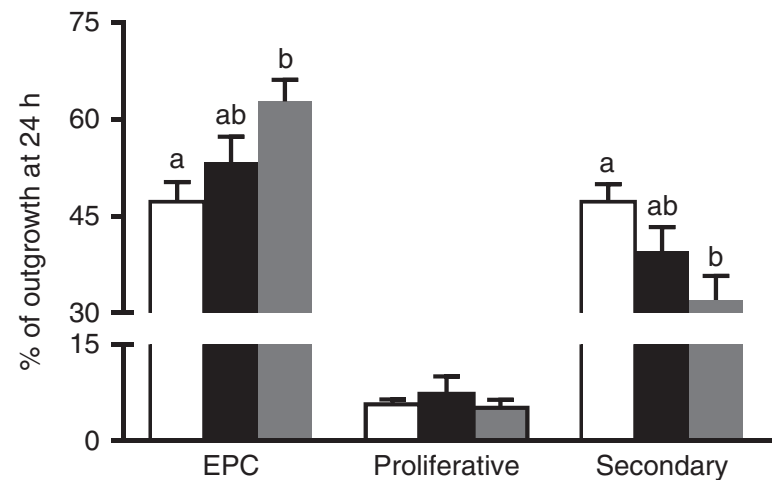

(b)

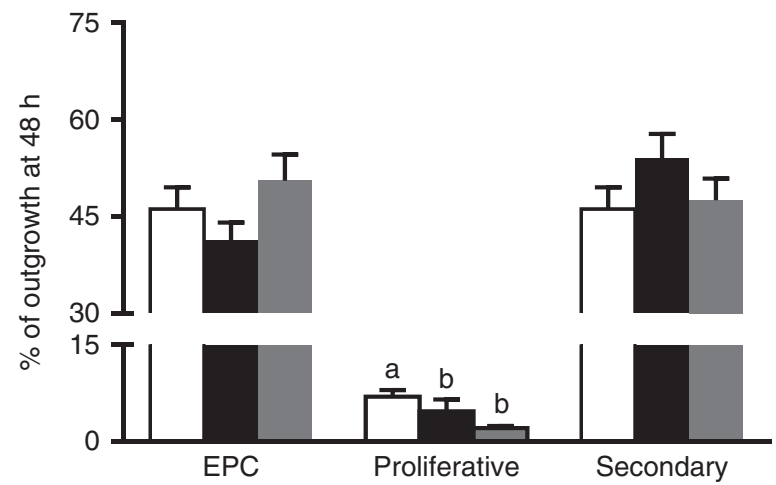

(c)

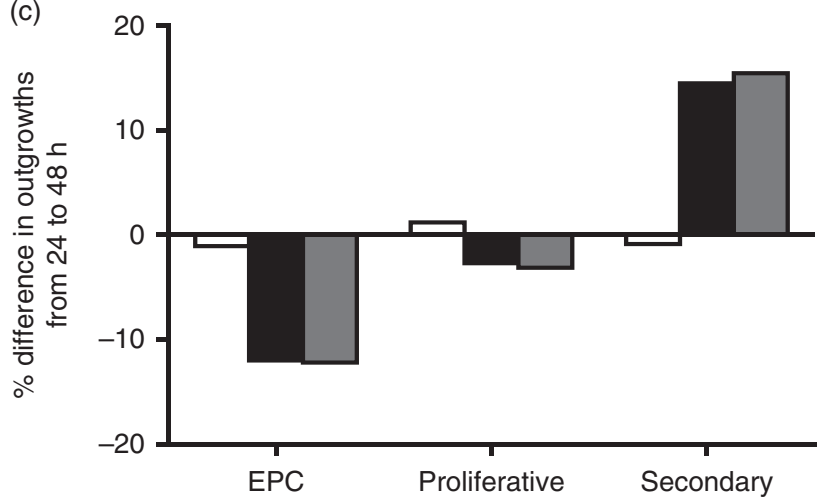

Figure 3 (a) Mean \% composition of NPD (white bars), LPD (black bars) and Emb-LPD (grey bars) explants grown on Matrigel-coated coverslips after 24 and (b) $48 \mathrm{~h}$. (c) Mean change in outgrowth composition between 24 and $48 \mathrm{~h}$ of culture. $n=10-14$ outgrowths per culture time and treatment group, taken from 6-8 separate females per diet. Error bars are S.E.M. Different letters denote statistical significance between groups at $P<0.05$.

weight at birth and throughout life of Emb-LPD-fed offspring. Therefore, to determine whether maternal diet influenced fetal development in the current study, we evaluated fetal growth at the same stage (E17) of gestation (Table 1). At the time of cull (E17), there was no significant difference in the mean litter size between treatment groups (NPD 12.86 \pm 0.95 , LPD $13.31 \pm 0.37$, Emb-LPD 11.15 $\pm 0.50 ; P>0.05)$. Concepti from EmbLPD-fed dams were significantly heavier than those from NPD- and LPD-fed dams $(P<0.05)$. Following dissection, Emb-LPD-fed fetuses also displayed elevated weight when compared with NPD- and LPD-fed fetuses $(P<0.05)$. Placentas from LPD-fed fetuses were significantly lighter than those of NPD- and Emb-LPD-fed fetuses $(P<0.05)$. The significantly increased Emb-LPD fetal weight and decreased LPD placental weight resulted in significantly increased fetal:placental ratios in both groups when compared with NPD-fed offspring. Although no difference in the mean yolk sac weight has been observed between dietary groups, Emb-LPDfed fetuses displayed a significantly elevated fetal: yolk sac ratio when compared with NPD- and LPD-fed fetuses $(P<0.01)$.

\section{E17 placental and yolk sac mTOR protein analysis}

Changes in placental size, function and mTORC1 activity in response to maternal gestational diet have been demonstrated previously (Rosario et al. 2011, Jansson et al. 2012). Therefore, we assessed the total and phosphorylated levels of the downstream targets of mTORC1, 4E-BP1 and S6 ribosomal protein, in placental and yolk sac tissues. 4E-BP1 acts as a translation repressor protein by binding to the translation initiation factor elF4E, an interaction inhibited by the phosphorylation of 4E-BP1 (Dowling et al. 2010). Conversely, the phosphorylation of S6 ribosomal protein, mediated by phosphorylated S6 kinase, results in the translation of terminal oligopyrimidine-dependent transcripts, encoding ribosomal proteins involved in cell cycle progression and translation regulation (Kim 2009). Analysis of total and phosphorylated 4E-BP1 and S6 ribosomal protein levels in offspring placental and yolk sac tissue revealed no significant differences between treatment groups (Fig. 5). However, a trend towards a reduced level of phosphorylated 4E-BP1 and a decrease in the ratio of phosphorylated to total 4E-BP1 in LPD placentas was observed when compared with NPD placentas ( $P=0.077$ and 0.099 respectively). Similarly, decreased levels of total and phosphorylated S6 ribosomal protein were observed in LPD placentas when compared with NPD and Emb-LPD placentas, though these did not reach significance. No differences were observed in the levels of 4E-BP1 or S6 ribosomal protein in offspring yolk sac tissue between dietary groups.

\section{Discussion}

Appropriate placental development and function plays a pivotal role in directing fetal growth, offspring phenotype and influencing adult predisposition to cardiovascular and metabolic disease risk (Vaughan et al. 2011). During early mammalian development, TGCs are fundamental for the establishment of maternal-fetal nutrient and gas exchange and in the formation of the placenta (Rossant \& Cross 2001). As the post-implantation EPC is considered 

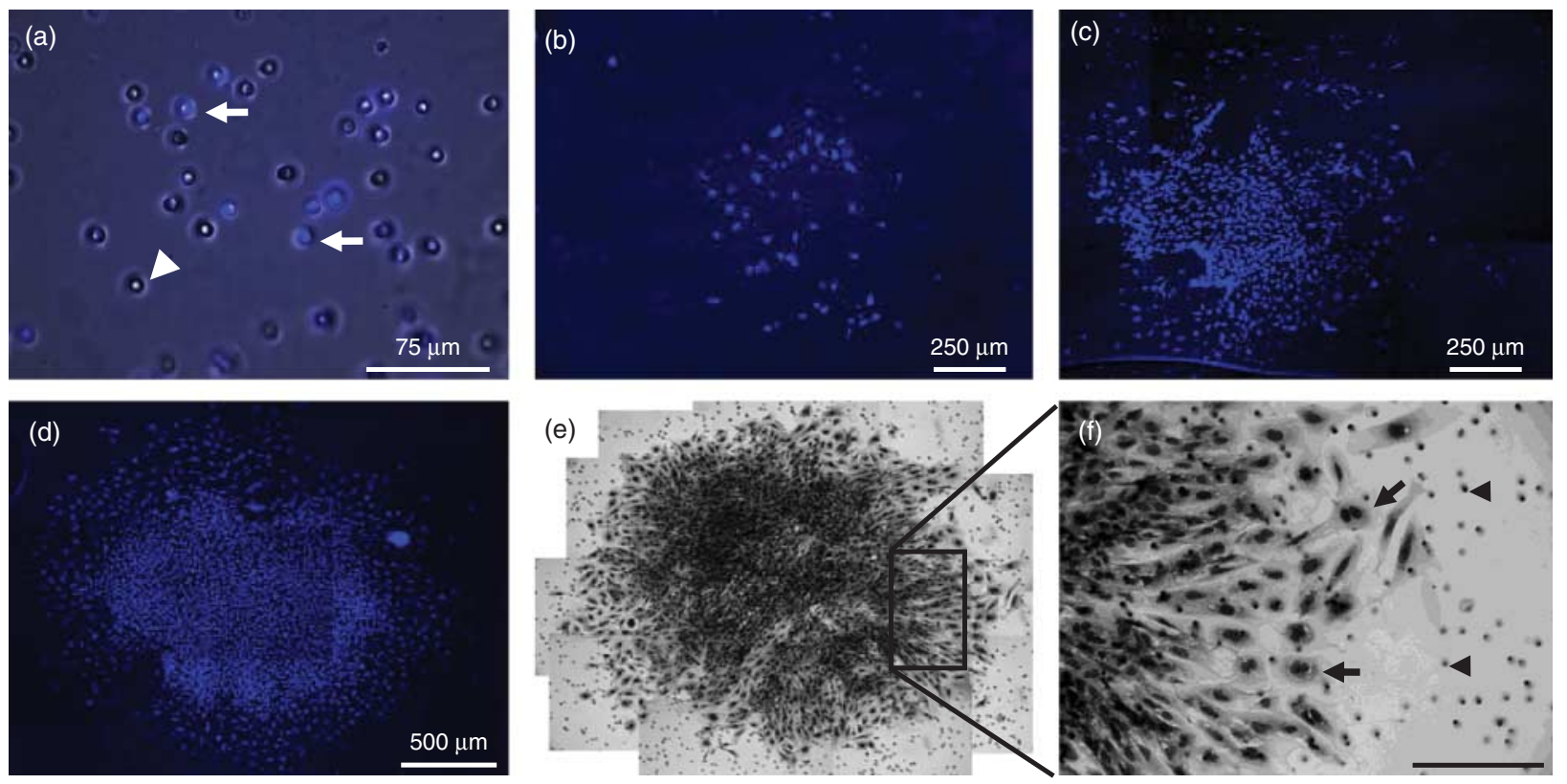

(g)

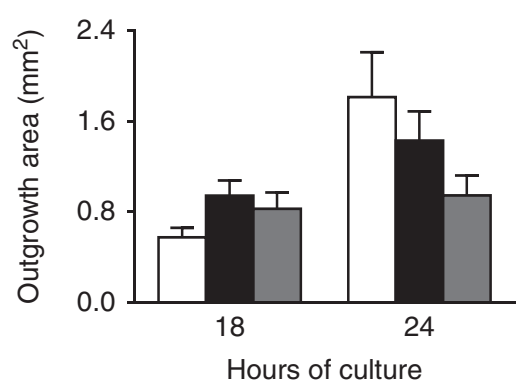

(j)

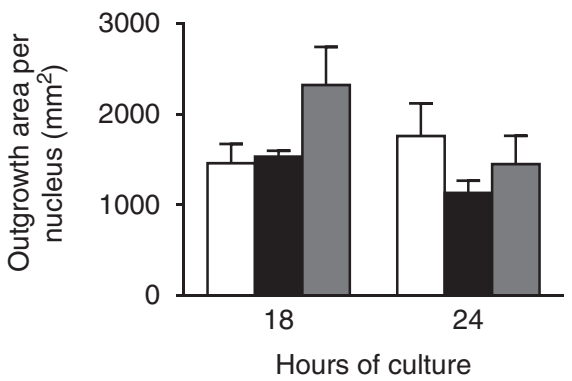

(h)

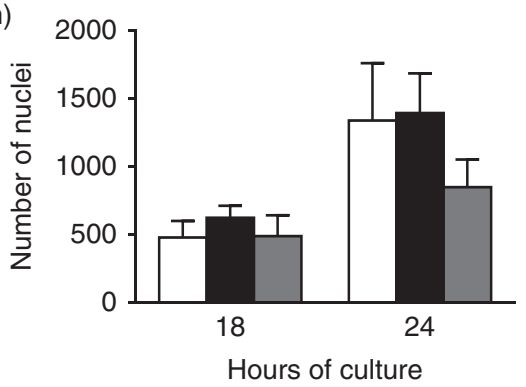

$(\mathrm{k})$

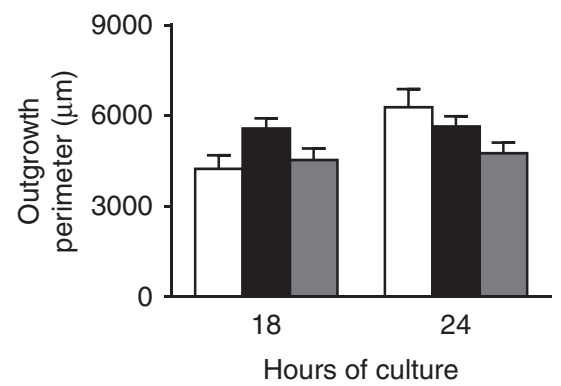

Figure 4 (a) High-magnification image of a DAPI-stained Transwell invasion chamber showing cells migrating (arrows) though the $8 \mu \mathrm{m}$ membrane pores (arrow head) after $6 \mathrm{~h}$ in culture. (b) Lower magnification image of a DAPI-stained Transwell invasion chamber after 6, (c) 12 and (d) $18 \mathrm{~h}$ of culture. (e) Haematoxylin-stained outgrowth after $18 \mathrm{~h}$ of culture. (f) Magnified section showing large, multinucleated peripheral cells (arrows) and $8 \mu \mathrm{m}$ pores (arrow heads). (g, h, i, j and k) Mean EPC outgrowth measurement from NPD (white bars), LPD (black bars) and Emb-LPD (grey bars) explants grown on Matrigel invasion transwell inserts after 18 and $24 \mathrm{~h} . n=6-7$ outgrowths per culture time and treatment group, taken from 6-7 separate females per diet. Error bars are S.E.M. Different letters denote statistical significance between groups at $P<0.05$.

the primary source of secondary TGCs, the in vitro culture of the EPC provides a system in which early placental dynamics can be assessed in response to maternal nutritional status. In this study, we investigated the impact of maternal low-protein dietary regimes on post-implantation trophoblast spreading phenotype and invasive capacity in vitro, fetal development and signalling through the nutritional sensing mTORC1 pathway in late gestation placenta and yolk sac tissues. We observed that maternal LPD enhanced the development and growth of EPC explants taken at E8.5, but that their invasive capacity remained unaltered when 
Table 1 Mean weights of concepti and fetal tissues from NPD-, LPDand Emb-LPD-fed mouse dams at day 17 of gestation.

\begin{tabular}{lccc}
\hline & \multicolumn{3}{c}{ Diet } \\
\cline { 2 - 4 } Tissue & NPD & LPD & Emb-LPD \\
\hline Conceptus (mg) & $1261.7 \pm 15.9^{\mathrm{a}}$ & $1227.6 \pm 18.5^{\mathrm{a}}$ & $1324.2 \pm 18.2^{\mathrm{b}}$ \\
Fetus (mg) & $941.2 \pm 8.6^{\mathrm{a}}$ & $943.4 \pm 9.4^{\mathrm{a}}$ & $1039.5 \pm 9.3^{\mathrm{b}}$ \\
Placenta (mg) & $169.2 \pm 2.5^{\mathrm{a}}$ & $150.1 \pm 1.9^{\mathrm{b}}$ & $159.7 \pm 2.2^{\mathrm{a}}$ \\
Yolk sac (mg) & $82.2 \pm 1.6$ & $87.6 \pm 1.6$ & $83.03 \pm 2.0$ \\
Fetal:Placental & $5.74 \pm 0.09^{\mathrm{a}}$ & $6.40 \pm 0.09^{\mathrm{b}}$ & $6.64 \pm 0.09^{\mathrm{b}}$ \\
Fetal:Yolk sac & $12.37 \pm 0.32^{\mathrm{a}}$ & $11.46 \pm 0.29^{\mathrm{a}}$ & $13.61 \pm 0.38^{\mathrm{b}}$ \\
\hline
\end{tabular}

$n=13-14$ dams per treatment group. Error values are S.E.M. Different letters denote statistical significance at $P<0.05$.

compared with explants taken from NPD- and Emb-LPDfed dams. In late gestation (E17), both maternal LPD and Emb-LPD treatments induced significant increases in the fetal:placental ratio, through reduced placental and enhanced fetal growth respectively. Signalling responses mediated by the mTORC1 pathway within the placenta and yolk sac tissues were similar across all treatment groups. These results expand our understanding of the early developmental mechanisms regulating fetal growth and development in response to maternal nutrition, providing novel insights into the programming of adult offspring health.

Mouse TGC differentiation has been characterised in detail, revealing a complex expression pattern of blood group antigens (B and Le-b/Le-y), integrins $\left(\alpha_{7}\right)$, hormones (PL-II, PTHrP) and transcription factors (AP-2 $2 \gamma$ ) both in vitro and in vivo (Centrella et al. 1989, Brown et al. 1993, Auman et al. 2002, El-Hashash et al. 2010). It has been demonstrated that cells with large nuclear area $\left(>300 \mu \mathrm{m}^{2}\right)$ located at the periphery of EPC outgrowths express PL-II and AP- $2 \gamma$, identifying these as secondary TGCs (El-Hashash \& Kimber 2004). In contrast, cells located immediately adjacent to the EPC have been shown to be proliferative stem cells (Scott et al. 2000). Therefore, using these pre-defined morphological (cell size, nuclear area, single or multi-layered) and locational (peripheral or adjacent to the EPC) characteristics, we quantitatively measured EPC explant outgrowth in vitro. EPC explants from all treatments groups outgrew in a radial fashion, retaining a central EPC mass surrounded by small, dense, round proliferative cells. At the periphery of each outgrowth were large, flat, multinucleated cells. Upon quantification of each of these outgrowth regions, we observed that EPC explants cultured from LPD-fed dams displayed significantly larger EPC and secondary TGC areas with increased numbers of secondary TGC nuclei when compared with NPD and Emb-LPD outgrowths after 24 and $48 \mathrm{~h}$. The increase in secondary TGC area appeared to be driven by increased rates of cell division, as indicated by an increased number of nuclei. However, a disproportionate increase in the number of large multi-nucleated secondary TGCs in LPD outgrowths cannot be discounted. In addition, the contribution of cells from the proliferative region was not assessed, and so an increase in cell allocation from this area cannot be dismissed. However, as no significant differences in mean proliferative TGC area were observed, the contribution to the increase in secondary TGC area may be small. Interestingly, we observed modest increases in EPC outgrowth in both NPD and Emb-LPD explants, differing from each other in EPC area (increased in Emb-LPD) and number of secondary TGCs (decreased in Emb-LPD) at $24 \mathrm{~h}$ only. Therefore, the up-regulation of EPC outgrowth phenotype in response to maternal LPD could provide a more metabolically efficient early gestation adaptive mechanism to promote enhanced embryonic-maternal vascular connection and maintain fetal development rather than increasing placental mass in late gestation. Indeed, changes in extraembryonic lineage allocation and spreading phenotype have been identified in blastocysts collected from LPD-fed mouse and rat dams (Kwong et al. 2000, Eckert et al. 2012). In addition, enhanced endocytotic and lysosomal activity within LPD blastocyst TE and embryoid body primitive endoderm has also been reported (Sun et al. 2014). Similar adaptive responses have been shown to occur in response to maternal diabetes, where enhanced rates of cell proliferation have been reported in EPCs and late gestation placentas collected from diabetic rat dams, while the culture of control dam EPCs in high glucose media increased rates of EPC spreading (Caluwaerts et al. 2000, Zorn et al. 2011).

To determine whether maternal diet affected outgrowth invasive capacity, we cultured isolated EPCs on Matrigel-coated porous membranes, allowing invasive cells to penetrate the gel and migrate to the lower surface. Matrigel assays have been used to characterise the invasive phenotype of human trophoblast cells (Sato et al. 2002, Xu et al. 2002, O'Brien et al. 2003), revealing differential invasive responses to a range of pro-inflammatory cytokines (Staff et al. 2000, Bauer et al. 2004, Pavan et al. 2004, Jovanovic et al. 2010). In mouse assays, TGCs have been shown to migrate through the insert ahead of smaller cell types (Hemberger et al. 2004). In vivo, altered patterns of trophoblast invasion have been reported in a rat model of maternal gestational obesity (Hayes et al. 2014). We observed that after $6-12 \mathrm{~h}$ in culture, relatively few cells had migrated to the lower surface of the membrane; however, after $36 \mathrm{~h}$ in culture, it was not possible to identify individual nuclei. Therefore, in order to characterise invasive and migratory phenotype, we assessed outgrowths after 18 and $24 \mathrm{~h}$ in culture. In contrast to our findings of EPC outgrowth phenotype on Matrigel-coated coverslips, we observed no difference in EPC explant invasive characteristics, with respect to maternal diet, when assessed using transwell inserts. This difference could be accounted for by the fact that, on coverslips, outgrowing cells can only migrate away radially and in a single plane from the central EPC; however, with transwell inserts, cells can disperse to the 
(a)

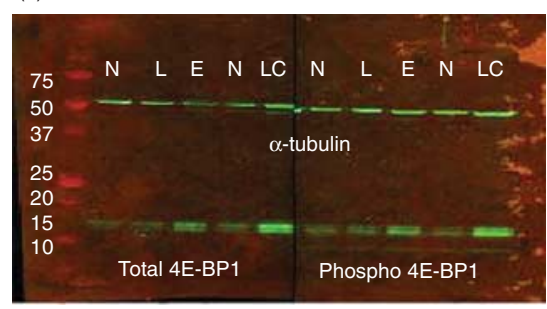

(c)

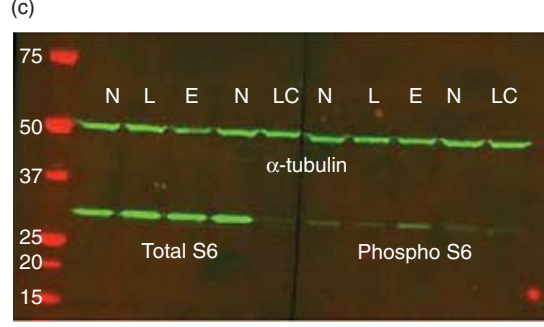

(b)

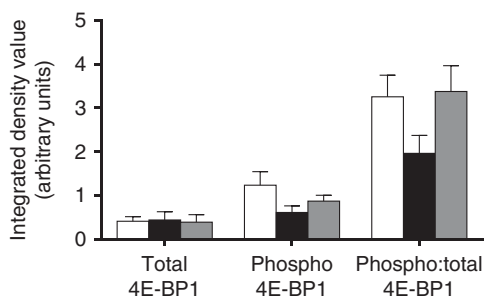

(d)

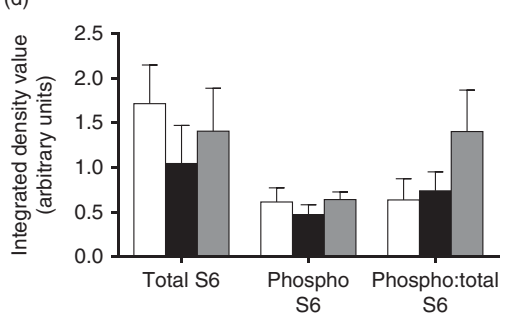

(f)
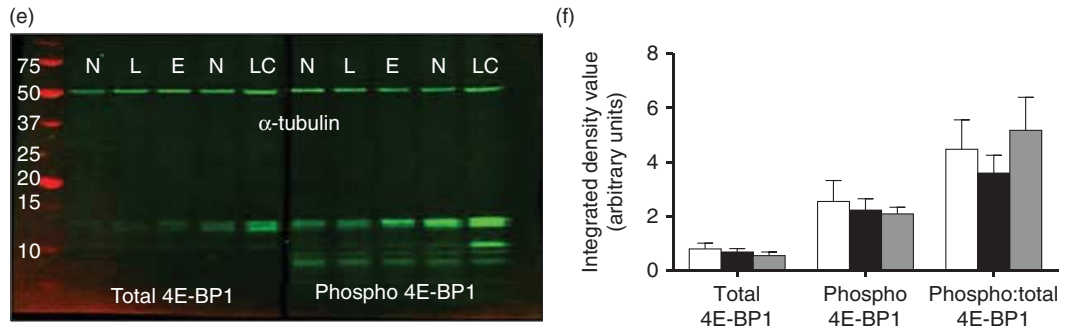

(g)

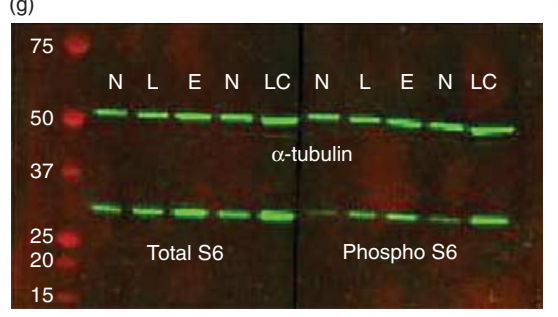

(h)

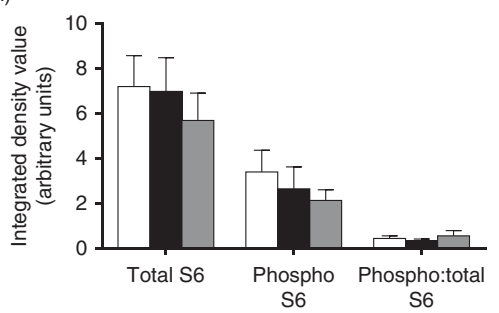

Figure 5 Representative immunoblots in E17 (a and c) placenta and (e and g) yolk sac tissues for mTORC1 downstream targets of total and phosphorylated (a and e) 4E-BP1 and (c and g) S6 protein levels and the reference protein $\alpha$-tubulin. Individual blot lanes include molecular weight markers (far left) and samples of NPD (N), LPD (L) and Emb-LPD (E) tissues (20 $\mu$ g total protein per lane) and loading control (LC). Mean integrated density values for total and phosphorylated levels of ( $b$ and $f$ ) 4E-BP1 and ( $d$ and h) S6 ribosomal protein in NPD (white bars), LPD (black bars) and Emb-LPD (grey bars) E17 (b and d) placental and ( $f$ and $h$ ) yolk sac tissues normalised to $\alpha$-tubulin. $n=8$ samples per treatment, all from separate litters. Error values are S.E.M. lower surface and migrate in any direction resulting in more diffuse outgrowth patterns. As it was only possible to analyse the lower surface of the transwell insert, it was not possible to assess the proportions of cells remaining on the upper surface, or which may still be migrating through the membrane. As such, for future studies increasing the number of analytical time points, assessing for specific markers of secondary TGCs, determination of gene and protein expression patterns within our outgrowths and/or assessing TGC development in vivo would all provide additional insights into the invasive characteristics of EPC outgrowths in response to maternal diet. In addition, as our outgrowths were cultured under ambient oxygen condition, as apposed to the more physiological low oxygen tension observed in utero, we cannot rule out potential confounding effects on TGC phenotype in response to this disparity. As such, future in vitro studies will employ conditions more physiologically relevant to mimic better those observed in utero.

It would be anticipated that any adaptive mechanisms initiated within LPD-fed pre-implantation embryos would also be induced within Emb-LPD-fed embryos. It was of interest to observe significant differences in outgrowth phenotype between LPD and Emb-LPD EPCs cultured on coverslips. Analysis of changes in outgrowth proportions over time did, however, reveal that LPD and Emb-LPD explants behaved in a similar manner, reducing the relative proportion of the EPC and increasing the relative proportion of secondary TGCs. This was in stark contrast to NPD outgrowths, which remained proportionally unchanged over the same period. As discussed earlier, we proposed that maternal LPD induces nutrient retrieval mechanisms within the extraembryonic lineages of the developing embryo. Therefore, maternal LPD may induce the blastocyst to invest disproportionately into 
the developing EPC after implantation. If the maternal diet remains sub-optimal, then the adaptive investment increases rates of cell division and nutrient uptake in order to maintain fetal development under conditions of reduced nutrient availability. However, when maternal NPD is restored at implantation (Emb-LPD), rates of trophoblast cell division are unaltered, but the programmed enhanced nutrient uptake adaptations result in increased fetal growth.

The development and size of the late gestation placenta is directly linked to nutrient transport capacity and fetal growth. Placental morphology, thickness, fetal-placental blood flow and the expression and function of nutrient transporters also influence fetal growth and development (Salafia et al. 2007). In addition to the placenta, the visceral yolk sac provides the developing fetus with a constant supply of amino acids through the breakdown of maternal proteins throughout gestation. Therefore, we determined whether changes in post-implantation trophoblast phenotype affected fetal, placental and yolk sac development in late gestation (E17). Our analysis revealed elevated fetal growth in offspring from EmbLPD-fed dams, reflecting our previous findings of elevated birth weight of Emb-LPD-fed offspring and of late gestation fetuses derived from LPD blastocysts transferred into NPD-fed dams (Watkins et al. 2008). In addition, we observed a significantly elevated fetal:yolk sac weight ratio in Emb-LPD-fed fetuses when compared with NPD- and LPD-fed fetuses. Maternal LPD in the mouse has been shown to enhance nutrient uptake activity within the blastocyst TE and embryoid body primitive endoderm (Sun et al. 2014) and elevate visceral yolk sac endocytosis in late gestation (Watkins et al. 2008). Therefore, the enhanced fetal growth observed in Emb-LPD-fed offspring could be programmed in part through increased delivery of essential nutrition by the yolk sac during gestation. Conversely, fetuses from LPDfed dams displayed comparable fetal weight but with a significantly reduced placental weight. The significantly increased Emb-LPD fetal weight, and decreased LPD placental weight resulted in significantly elevated fetal:placental weight ratios in both LPD- and EmbLPD-fed fetuses. The fetal:placental ratio is conceived as an indicator of placental nutrient transport capacity, with an increased ratio considered as a sign of enhanced placental efficiency (Fowden et al. 2009). As discussed earlier in this study, in response to maintained maternal LPD, it may be more metabolically favourable to establish placentation earlier and minimise placental growth in later gestation when maternal resources are constrained. However, this adaptation may then result in altered placental function and transport, having important consequences for offspring growth and adult health. Maternal LPD in rats has been shown to result in the down-regulation of placental amino acid transporters ahead of development of intrauterine fetal growth restriction (Malandro et al. 1996, Jansson et al. 2006).
Sferruzzi-Perri et al. (2013) demonstrated that a maternal obesogenic diet reduced fetal and placental growth, but enhanced placental glucose and amino acid transport, as well as expression of fatty acid transporters and metabolic signalling pathway mediators. The molecular regulation of placental nutrient transport is believed to be coordinated through mTORC1, acting as a central nutrient sensor orchestrating fetal demand with maternal supply (Jansson et al. 2012). Maternal gestational nutrient restriction has been shown to decrease placental mTORC1 activity in the baboon (Kavitha et al. 2014) and human IUGR pregnancies (Roos et al. 2007, Yung et al. 2008), and in response to rodent LPD (Jansson et al. 2006, Rosario et al. 2011). In addition, circulating levels of insulin and branched chain amino acids, activators of mTORC1, are altered in models of maternal dietary induced obesity (Gaccioli et al. 2013, Lager et al. 2014). Therefore, we investigated the expression of key downstream targets of mTORC1 and their phosphorylation status in placentas from LPD- and Emb-LPD-fed dams. Herein, we observed minimal impacts of maternal diet on signalling through the mTORC1 pathway as assessed by the total and phosphorylated levels of the initiation factor elF4E and S6 ribosomal protein in either placental or yolk sac tissues. While significant changes in maternal metabolite and growth factor levels have been shown at the time of implantation in response to maternal LPD (Kwong et al. 2000, Eckert et al. 2012), the demonstration of late gestation maternal autophagy in rat dams fed LPD (Wang et al. 2014) raises the possibility that maternal nutritional status stabilises after several days of LPD, resulting in the similar levels of $\mathrm{mTOC}_{1}$ signalling observed in this study. In addition, as physiological exchange between maternal and fetal circulations occurs within the labyrinthine zone of the placenta, our analysis of mTOR signalling within the whole placenta may have masked zone-specific differences. Finally, the placenta may augment other mechanisms independently of mTOR to modify nutrient transport, thus resulting in similar levels of mTOR signalling, but still affecting fetal development. For example, in nutrient-restricted sheep at mid-gestation, a reduction in fetal growth is observed with increased AMPK and ERK1/2 activity but with no change in mTOR and Akt signalling (Ma et al. 2011).

Taken together, our results indicate that maternal LPD programmes post-implantation trophoblast growth and phenotype in order to maintain embryonic and fetal development within a sub-optimal nutritional environment. In late gestation, the maintenance of maternal LPD results in a significantly smaller placenta but with comparable fetal weight, suggestive of enhanced placental efficiency and transport. However, these adaptive mechanisms, while supporting offspring development to birth and reproductive age, become maladaptive and predispose offspring to adultonset disease (Watkins et al. 2008). In contrast, maternal Emb-LPD does not affect early post-implantation trophoblast spreading and cell number, due potentially to the 
restoration of maternal nutrition to optimal (NPD) levels after implantation. However, the induction of enhanced nutrient uptake capacity, programmed within the blastocyst in response to preimplantation LPD, results in elevated fetal growth in late gestation, also inducing adult cardiovascular and metabolic disease (Watkins et al. 2008). These data add new insights into the sensitivity of the developing embryo in response to maternal nutrition at the time of embryo implantation, for the programming of offspring growth and subsequent health.

\section{Declaration of interest}

The authors declare that there is no conflict of interest that could be perceived as prejudicing the impartiality of the research reported.

\section{Funding}

This work was supported through awards from the Biotechnology and Biological Sciences Research Council (BB/F007450/1; BB/I001840/1) to T P Fleming.

\section{Acknowledgements}

The authors thank staff from the University of Southampton Biomedical Research Facility for animal provision and maintenance.

\section{References}

Adamson SL, Lu Y, Whiteley KJ, Holmyard D, Hemberger M, Pfarrer C \& Cross JC 2002 Interactions between trophoblast cells and the maternal and fetal circulation in the mouse placenta. Developmental Biology 250 358-373. (doi:10.1006/dbio.2002.0773)

Armitage JA, Khan IY, Taylor PD, Nathanielsz PW \& Poston L 2004 Developmental programming of the metabolic syndrome by maternal nutritional imbalance: how strong is the evidence from experimental models in mammals? Journal of Physiology 561 355-377. (doi:10.1113/ jphysiol.2004.072009)

Auman HJ, Nottoli T, Lakiza O, Winger Q, Donaldson S \& Williams T 2002 Transcription factor AP- $2 \gamma$ is essential in the extra-embryonic lineages for early postimplantation development. Development 129 2733-2747.

Bauer S, Pollheimer J, Hartmann J, Husslein P, Aplin JD \& Knofler M 2004 Tumor necrosis factor- $\alpha$ inhibits trophoblast migration through elevation of plasminogen activator inhibitor-1 in first-trimester villous explant cultures. Journal of Clinical Endocrinology and Metabolism 89 812-822. (doi:10.1210/jc.2003-031351)

Bevilacqua EM \& Abrahamsohn PA 1988 Ultrastructure of trophoblast giant cell transformation during the invasive stage of implantation of the mouse embryo. Journal of Morphology 198 341-351. (doi:10.1002/jmor.1051980308)

Brown DG, Warren VN, Pahlsson P \& Kimber SJ 1993 Carbohydrate antigen expression in murine embryonic stem cells and embryos. I. Lacto and neo-lacto determinants. Histochemical Journal 25 452-463. (doi:10. 1007/BF00157810)

Caluwaerts S, Pijnenborg R, Luyten C \& Van Assche FA 2000 Growth characteristics of diabetic rat ectoplacental cones in vivo and in vitro. Diabetologia 43 939-945. (doi:10.1007/s001250051473)

Centrella M, Canalis E, McCarthy TL, Stewart AF, Orloff JJ \& Insogna KL 1989 Parathyroid hormone-related protein modulates the effect of transforming growth factor- $\beta$ on deoxyribonucleic acid and collagen synthesis in fetal rat bone cells. Endocrinology 125 199-208. (doi:10. 1210/endo-125-1-199)
Chen PY, Ganguly A, Rubbi L, Orozco LD, Morselli M, Ashraf D, Jaroszewicz A, Feng S, Jacobsen SE, Nakano A et al. 2013 Intrauterine calorie restriction affects placental DNA methylation and gene expression. Physiological Genomics 45 565-576. (doi:10.1152/physiolgenomics.00034.2013)

Coan PM, Vaughan OR, McCarthy J, Mactier C, Burton GJ, Constancia M \& Fowden AL 2011 Dietary composition programmes placental phenotype in mice. Journal of Physiology 589 3659-3670. (doi:10.1113/jphysiol. 2011.208629)

Dowling RJ, Topisirovic I, Fonseca BD \& Sonenberg N 2010 Dissecting the role of mTOR: lessons from mTOR inhibitors. Biochimica et Biophysica Acta 1804 433-439. (doi:10.1016/j.bbapap.2009.12.001)

Eckert JJ, Porter R, Watkins AJ, Burt E, Brooks S, Leese HJ, Humpherson PG, Cameron IT \& Fleming TP 2012 Metabolic induction and early responses of mouse blastocyst developmental programming following maternal low protein diet affecting life-long health. PLOS ONE 7 e52791. (doi:10.1371/journal.pone.0052791)

El-Hashash AH \& Kimber SJ 2004 Trophoblast differentiation in vitro: establishment and characterisation of a serum-free culture model for murine secondary trophoblast giant cells. Reproduction 128 53-71. (doi:10.1530/rep.1.00149)

El-Hashash AH, Esbrit P \& Kimber SJ 2005 PTHrP promotes murine secondary trophoblast giant cell differentiation through induction of endocycle, upregulation of giant-cell-promoting transcription factors and suppression of other trophoblast cell types. Differentiation 73 154-174. (doi:10.1111/j.1432-0436.2005.00013.x)

El-Hashash AH, Warburton D \& Kimber SJ 2010 Genes and signals regulating murine trophoblast cell development. Mechanisms of Development 127 1-20. (doi:10.1016/j.mod.2009.09.004)

Fowden AL, Sferruzzi-Perri AN, Coan PM, Constancia M \& Burton GJ 2009 Placental efficiency and adaptation: endocrine regulation. Journal of Physiology 587 3459-3472. (doi:10.1113/jphysiol.2009.173013)

Gabory A, Ferry L, Fajardy I, Jouneau L, Gothie JD, Vige A, Fleur C, Mayeur S, Gallou-Kabani C, Gross MS et al. 2012 Maternal diets trigger sex-specific divergent trajectories of gene expression and epigenetic systems in mouse placenta. PLOS ONE 7 e47986. (doi:10.1371/journal. pone.0047986)

Gaccioli F, White V, Capobianco E, Powell TL, Jawerbaum A \& Jansson T 2013 Maternal overweight induced by a diet with high content of saturated fat activates placental mTOR and elF $2 \alpha$ signaling and increases fetal growth in rats. Biology of Reproduction 89 96. (doi:10.1095/ biolreprod.113.109702)

Georgiades P, Ferguson-Smith AC \& Burton GJ 2002 Comparative developmental anatomy of the murine and human definitive placentae. Placenta 23 3-19. (doi:10.1053/plac.2001.0738)

Gheorghe CP, Goyal R, Holweger JD \& Longo LD 2009 Placental gene expression responses to maternal protein restriction in the mouse. Placenta 30 411-417. (doi:10.1016/j.placenta.2009.03.002)

Hanson MA \& Gluckman PD 2014 Early developmental conditioning of later health and disease: physiology or pathophysiology? Physiological Reviews 94 1027-1076. (doi:10.1152/physrev.00029.2013)

Hayes EK, Tessier DR, Percival ME, Holloway AC, Petrik JJ, Gruslin A \& Raha S 2014 Trophoblast invasion and blood vessel remodeling are altered in a rat model of lifelong maternal obesity. Reproductive Sciences 21 648-657. (doi:10.1177/1933719113508815)

Hemberger M, Hughes M \& Cross JC 2004 Trophoblast stem cells differentiate in vitro into invasive trophoblast giant cells. Developmental Biology 271 362-371. (doi:10.1016/j.ydbio.2004.03.040)

Jansson N, Pettersson J, Haafiz A, Ericsson A, Palmberg I, Tranberg M, Ganapathy V, Powell TL \& Jansson T 2006 Down-regulation of placental transport of amino acids precedes the development of intrauterine growth restriction in rats fed a low protein diet. Journal of Physiology 576 935-946.

Jansson T, Aye IL \& Goberdhan DC 2012 The emerging role of mTORC1 signaling in placental nutrient-sensing. Placenta 33 (Suppl 2) e23-e29. (doi:10.1016/j.placenta.2012.05.010)

Jovanovic M, Stefanoska I, Radojcic L \& Vicovac L 2010 Interleukin-8 (CXCL8) stimulates trophoblast cell migration and invasion by increasing levels of matrix metalloproteinase (MMP)2 and MMP9 and integrins $\alpha 5$ and $\beta 1$. Reproduction 139 789-798. (doi:10.1530/REP-09-0341)

Kavitha JV, Rosario FJ, Nijland MJ, McDonald TJ, Wu G, Kanai Y, Powell TL, Nathanielsz PW \& Jansson T 2014 Down-regulation of placental mTOR, 
insulin/IGF-I signaling, and nutrient transporters in response to maternal nutrient restriction in the baboon. FASEB Journal 28 1294-1305. (doi:10. 1096/fj.13-242271)

Kim E 2009 Mechanisms of amino acid sensing in mTOR signaling pathway. Nutrition Research and Practice 3 64-71. (doi:10.4162/nrp.2009.3.1.64)

Kim DW, Young SL, Grattan DR \& Jasoni CL 2014 Obesity during pregnancy disrupts placental morphology, cell proliferation, and inflammation in a sex-specific manner across gestation in the mouse. Biology of Reproduction 90 130. (doi:10.1095/biolreprod.113.117259)

Kwong WY, Wild AE, Roberts P, Willis AC \& Fleming TP 2000 Maternal undernutrition during the preimplantation period of rat development causes blastocyst abnormalities and programming of postnatal hypertension. Development 127 4195-4202.

Lager S, Samulesson AM, Taylor PD, Poston L, Powell TL \& Jansson T 2014 Diet-induced obesity in mice reduces placental efficiency and inhibits placental mTOR signaling. Physiological Reports 2 e00242. (doi:10.1002/phy2.242)

Ma Y, Zhu MJ, Uthlaut AB, Nijland MJ, Nathanielsz PW, Hess BW \& Ford SP 2011 Upregulation of growth signaling and nutrient transporters in cotyledons of early to mid-gestational nutrient restricted ewes. Placenta 32 255-263. (doi:10.1016/j.placenta.2011.01.007)

Malandro MS, Beveridge MJ, Kilberg MS \& Novak DA 1996 Effect of lowprotein diet-induced intrauterine growth retardation on rat placental amino acid transport. American Journal of Physiology 271 C295-C303.

O'Brien PJ, Koi H, Parry S, Brass LF, Strauss JF III, Wang LP, Tomaszewski JE \& Christenson LK 2003 Thrombin receptors and protease-activated receptor-2 in human placentation: receptor activation mediates extravillous trophoblast invasion in vitro. American Journal of Pathology 163 1245-1254. (doi:10.1016/S0002-9440(10)63484-0)

Pavan L, Hermouet A, Tsatsaris V, Therond P, Sawamura T, Evain-Brion D \& Fournier T 2004 Lipids from oxidized low-density lipoprotein modulate human trophoblast invasion: involvement of nuclear liver $\mathrm{X}$ receptors. Endocrinology 145 4583-4591. (doi:10.1210/en.2003-1747)

Roos S, Jansson N, Palmberg I, Saljo K, Powell TL \& Jansson T 2007 Mammalian target of rapamycin in the human placenta regulates leucine transport and is down-regulated in restricted fetal growth. Journal of Physiology 582 449-459. (doi:10.1113/jphysiol.2007.129676)

Rosario FJ, Jansson N, Kanai Y, Prasad PD, Powell TL \& Jansson T 2011 Maternal protein restriction in the rat inhibits placental insulin, mTOR, and STAT3 signaling and down-regulates placental amino acid transporters. Endocrinology 152 1119-1129. (doi:10.1210/en.2010-1153)

Rossant J \& Cross JC 2001 Placental development: lessons from mouse mutants. Nature Reviews. Genetics 2 538-548. (doi:10.1038/35080570)

Salafia CM, Zhang J, Miller RK, Charles AK, Shrout P \& Sun W 2007 Placental growth patterns affect birth weight for given placental weight. Birth Defects Research. Part A, Clinical and Molecular Teratology 79 281-288. (doi:10.1002/bdra.20345)

Sato Y, Fujiwara H, Higuchi T, Yoshioka S, Tatsumi K, Maeda M \& Fujii S 2002 Involvement of dipeptidyl peptidase IV in extravillous trophoblast invasion and differentiation. Journal of Clinical Endocrinology and Metabolism 87 4287-4296. (doi:10.1210/jc.2002-020038)

Scott IC, Anson-Cartwright L, Riley P, Reda D \& Cross JC 2000 The HAND1 basic helix-loop-helix transcription factor regulates trophoblast differentiation via multiple mechanisms. Molecular and Cellular Biology 20 530-541. (doi:10.1128/MCB.20.2.530-541.2000)

Sferruzzi-Perri AN, Vaughan OR, Haro M, Cooper WN, Musial B, Charalambous M, Pestana D, Ayyar S, Ferguson-Smith AC, Burton GJ et al. 2013 An obesogenic diet during mouse pregnancy modifies maternal nutrient partitioning and the fetal growth trajectory. FASEB Journal 27 3928-3937. (doi:10.1096/fj.13-234823)
Simmons DG \& Cross JC 2005 Determinants of trophoblast lineage and cell subtype specification in the mouse placenta. Developmental Biology 284 12-24. (doi:10.1016/j.ydbio.2005.05.010)

Simmons DG, Fortier AL \& Cross JC 2007 Diverse subtypes and developmental origins of trophoblast giant cells in the mouse placenta. Developmental Biology 304 567-578. (doi:10.1016/j.ydbio.2007.01. 009)

Soares MJ, Konno T \& Alam SM 2007 The prolactin family: effectors of pregnancy-dependent adaptations. Trends in Endocrinology and Metabolism 18 114-121. (doi:10.1016/j.tem.2007.02.005)

Staff AC, Ranheim T, Henriksen T \& Halvorsen B 2000 8-Iso-prostaglandin $\mathrm{f}(2 \alpha)$ reduces trophoblast invasion and matrix metalloproteinase activity. Hypertension 35 1307-1313. (doi:10.1161/01.HYP.35.6.1307)

Sun C, Velazquez MA, Marfy-Smith S, Sheth B, Cox A, Johnston DA, Smyth N \& Fleming TP 2014 Mouse early extra-embryonic lineages activate compensatory endocytosis in response to poor maternal nutrition. Development 141 1140-1150. (doi:10.1242/dev.103952)

Vaughan OR, Sferruzzi-Perri AN, Coan PM \& Fowden AL 2011 Environmental regulation of placental phenotype: implications for fetal growth. Reproduction, Fertility, and Development 24 80-96. (doi:10.1071/RD11909)

Wang H, Zhou D \& Pan Y-X 2014 Low protein diet during gestation and lactation induces autophagy-related gene LC3 in the liver of rat dams (737.5). FASEB Journal 28. (http://www.fasebj.org/citmgr?gca= fasebj\%3B28\%2F1_Supplement\%2F737.5)

Watkins AJ, Ursell E, Panton R, Papenbrock T, Hollis L, Cunningham C, Wilkins A, Perry VH, Sheth B, Kwong WY et al. 2008 Adaptive responses by mouse early embryos to maternal diet protect fetal growth but predispose to adult onset disease. Biology of Reproduction 78 299-306. (doi:10.1095/biolreprod.107.064220)

Watkins AJ, Lucas ES, Torrens C, Cleal JK, Green L, Osmond C, Eckert JJ, Gray WP, Hanson MA \& Fleming TP 2010 Maternal low-protein diet during mouse pre-implantation development induces vascular dysfunction and altered renin-angiotensin-system homeostasis in the offspring. British Journal of Nutrition 103 1762-1770. (doi:10.1017/ S0007114509993783)

Watkins AJ, Lucas ES, Wilkins A, Cagampang FR \& Fleming TP 2011 Maternal periconceptional and gestational low protein diet affects mouse offspring growth, cardiovascular and adipose phenotype at 1 year of age. PLOS ONE 6 e28745. (doi:10.1371/journal.pone.0028745)

Xu G, Guimond MJ, Chakraborty C \& Lala PK 2002 Control of proliferation, migration, and invasiveness of human extravillous trophoblast by decorin, a decidual product. Biology of Reproduction 67 681-689. (doi:10.1095/biolreprod67.2.681)

Yung HW, Calabrese S, Hynx D, Hemmings BA, Cetin I, CharnockJones DS \& Burton GJ 2008 Evidence of placental translation inhibition and endoplasmic reticulum stress in the etiology of human intrauterine growth restriction. American Journal of Pathology 173 451-462. (doi:10.2353/ajpath.2008.071193)

Zorn TM, Zuniga M, Madrid E, Tostes R, Fortes Z, Giachini F \& San Martin S 2011 Maternal diabetes affects cell proliferation in developing rat placenta. Histology and Histopathology 26 1049-1056.

Received 19 December 2014

First decision 19 January 2015

Revised manuscript received 2 March 2015

Accepted 9 March 2015 